\title{
EFEITO DE INSETICIDAS SOBRE A JOANINHA CYCLONEDA SANGUINEA L. (COLEOPTERA, COCCINELLIDAE) E SOBRE O PULGÃO APHIS GOSSYPII GLOVER (HEMIPTERA, APHIDIDAE) EM ALGODOEIRO
}

\author{
J.R. Scarpellini'; ${ }^{1}$ D.J. de Andrade ${ }^{2}$
}

${ }^{1}$ Apta Regional Centro Leste, Rod. Anel Viário, km 321, CEP 14032-970, Ribeirão Preto, SP, Brasil. E-mail: jrscarpellini@apta.sp.gov.br

\section{RESUMO}

As joaninhas são reconhecidas como importantes inimigos naturais de pragas, como pulgões e cochonilhas, apresentando excelente capacidade de dispersão no campo e podem ser favorecidas com ambientes para refúgio e seletividade dos defensivos agrícolas. O objetivo deste trabalho foi avaliar o efeito de inseticidas sobre a joaninha Cycloneda sanguinea e sobre sua presa, o pulgão Aphis gossypii, na cultura do algodão. Foram estudados os seguintes tratamentos (expressos em quantidade de produto comercial por hectare - p.c./ha): flonicamida 500 WG a 50, 80 e 150 g, thiamethoxam 250 WG a 200 g, acetamiprido 200 PS a 150 g, imidacloprido 200 SC a $250 \mathrm{~mL}$ e testemunha sem aplicação. A avaliação da seletividade fisiológica a $C$. sanguinea foi observada em laboratório e campo, avaliando-se o efeito dos inseticidas aplicados diretamente e indiretamente sobre C. sanguinea. Avaliou-se o número de joaninhas e pulgões sobreviventes após a aplicação. Com base nos resultados pode-se concluir que o inseticida flonicamida apresentou maior seletividade a $C$. sanguinea em relação aos demais inseticidas. Verificou-se que todos os tratamentos foram eficientes no controle do pulgão e não diferiram estatisticamente entre si.

PALAVRAS-CHAVES: Gossypium hirsutum, seletividade, controle biológico e inimigo natural.

\begin{abstract}
THE EFFECT OF INSECTICIDESON THE LADY BEETLE CYCLONEDA SANGUINEA L. (COLEOPTERA, COCCINELLIDAE) ANDONTHEAPHID APHIS GOSSYPIIGLOVER(HEMIPTERA, APHIDIDAE) ON COTTON PLANTS. The ladybugs are recognized as important natural enemies of pests such as aphids and scale insects, showing excellent dispersibility in the field and may be favored with environments for refuge and by pesticide selectivity. The goal of this trial was to study the toxicity of insecticides on the predatory coccinellid Cycloneda sanguinea and on its prey, the aphid Aphis gossypii, in the cotton crop. The treatments (expressed in commercial product per hectare) were: flonicamid 500 WG at 50, 80 and $150 \mathrm{~g}$, thiamethoxam 250 WG at $200 \mathrm{~g}$, acetamiprid 200 PS at $150 \mathrm{~g}$, imidacloprid 200 SC at $250 \mathrm{~mL}$, and a control treatment without application. The evaluation of physiological selectivity was made in the laboratory and in the field, observing the effect of insecticides applied directly and indirectly on C. sanguine. The number of surviving aphids and lady beetles was evaluated before and after the application. Based on the results obtained in the laboratory and field, it was concluded that flonicamid presented the highest selectivity to $C$. sanguinea among the insecticides tested. All treatments were effective for aphid control and there were no significant differences among them.
\end{abstract}

KEY WORDS: Gossypium hirsutum, selectivity, biological control, and natural enemy.

\section{INTRODUÇÃO}

Entre as principais pragas do algodoeiro, destacase o pulgão Aphis gossypii Glover (Hemiptera, Aphididae) que, além de inocular vírus causadores de doenças, produz secreções adocicadas, conhecida como honeydew, que contribui para o desenvolvi- mento de fungos do gênero Capnodium, popularmente chamados de fumagina que prejudicam a respiração e a fotossíntese das folhas. Quando ocorre ataque severo do A. gossypii na fase final da cultura, ocorre depreciação da qualidade da fibra, pela contaminação excessiva de honeydew, afetando sua utilização industrial (SANTOS, 1999).

${ }^{2}$ Universidade Estadual Paulista, Faculdade de Ciências Agrárias e Veterinárias, Jaboticabal, SP, Brasil. 
Em relação ao controle biológico na cultura do algodoeiro, as joaninhas pertencentes às espécies Cycloneda sanguinea e Hippodamia convergens destacam-se como importantes inimigos naturais, contribuindo eficientemente para controle do pulgão $A$. gossypii. Segundo HodeK (1967), os coccinelídeos predadores possuem grandecapacidade de dispersãono campo e de busca da presa, por isso são considerados agentes importantes no controle biológico de pragas.

BoIÇAJUNIOR et al. (2004) observaram em condições de casa-de-vegetação que adultos de $C$. sanguinea reduziram em $93,5 \%$ a população de $A$. gossypii em apenas dois dias. Todavia, em campo observa-se que nem sempre as joaninhas conseguem manter o nível populacional de pulgões abaixo do nível de dano econômico, tornandonecessárias intervençõesquímicas, utilizando-se preferencialmente os agrotóxicos mais seletivos possíveis aos inimigos naturais.

SOARES; BusOli (2000) verificaram que o inseticida fipronil provoca impacto menor em população de $C$. sanguinea do que endosulfan, nas primeiras vinte e quatro horas após a aplicação. SCARPELLINI (2008) constatou que a recuperação da população de predadores, após aplicações de carbosulfan e do acetamiprido, ocorreu sete dias após a aplicação.

No controle do A. gossypii, além do controle químico imprescindível quando da utilização de cultivares susceptíveis a viroses, tem sido recomendado outros sistemas de manejo queutilizem os métodos de controle biológico e cultural (ISKBER; COPLAND, 2002).

Diante deste contexto, pesquisas sobre os efeitos colaterais dos agrotóxicos em organismos benéficos têm se tornado obrigatórias em diversos países, fazendo com que se estabeleçam linhas de ação internacionalmente aprovadas, oferecendo aos produtores e técnicos informações para emprego adequado desses insumos (HASsAn et al., 1994). No Brasil, praticamente não existe padronização de procedimentos de pesquisa para avaliar seletividade dos agrotóxicos sobre a maioria dos organismos benéficos nos diferentes cultivos, bem como não é levado em consideração o efeito colateral dos defensivos nos artrópodes predadores presentes no algodoeiro, o que pode causar surtos de pragas secundárias, ressurgência e resistência das pragas (MAIA et al., 2001).

O presente trabalho objetivou avaliar o efeito de inseticidas sobre o coccinelídeo C. sanguinea e sobre sua presa o pulgão $A$. gossypii.

\section{MATERIAL E MÉTODOS}

A pesquisa foi realizada na Estação Experimental da Agência Paulista de Tecnologia dos Agronegócios (APTA) do Centro Leste, localizada no Município de Ribeirão Preto, SP, Brasil, no período de 18/3/ 2004 a 23/2/2005.

Para a obtenção da população-teste, inicialmente, foram coletadas joaninhas da espécie C. sanguinea em área localizada na Estação Experimental da APTA, contendo plantas de losna-branca (Parthenium hysterophorus) intensamente infestadas por pulgões; conforme metodologia proposta por SOARES; BUSOLI (2000). As joaninhas capturadas foram colocadas em tubos de vidro $(2,5 \mathrm{~cm}$ de diâmetro $\mathrm{x} 10 \mathrm{~cm}$ de altura), fechados com algodão e acondicionados no laboratório. Diariamente, pulgões da espécie $A$. gossypii foram colocados no interior dos tubos para servirem de alimento às joaninhas; aos pulgões, gotículas de mel foram oferecidas como alimento.

Experimento em laboratório: $\mathrm{O}$ delineamento adotado foi o inteiramente casualizado, com sete tratamentos equatro repetições, expressos em g ou mL de p.c./100 L de água (Tabela 1). Cada parcela experimental tinha 10 tubos de vidro $(2,5 \mathrm{~cm}$ de diâmetro $x$ $10 \mathrm{~cm}$ de altura), totalizando 40 tubos por tratamento.

No estudo para avaliar o efeito da exposição direta aos inseticidas foi colocada, no interior de cada tubo, uma joaninha C. sanguinea, procedente da criaçãoestoque e, na sequência, aplicou-se os produtos na parteinterna dos tubos, até um completo molhamento da parede do tubo, com auxílio de um pulverizador manual. Após a aplicação, cada tubo foi fechado com algodãoe mantido em condições normais de temperatura e umidade em caixas porta-tubo. Diariamente, foram oferecidas presas (pulgões) às joaninhas.

Tabela 1 - Relação dos tratamentos utilizados nos experimentos de avaliação do efeito de inseticidas sobre C. sanguinea na cultura do algodão.

\begin{tabular}{|c|c|c|c|c|}
\hline \multirow{2}{*}{ Tratamentos } & \multirow{2}{*}{ Ingrediente ativo } & \multirow{2}{*}{ Nome comercial } & \multicolumn{2}{|c|}{ Dosagens } \\
\hline & & & mL p.c./ha & g ou $\mathrm{mL}$ p.c./ha \\
\hline 1 & Flonicamida & Turbine 500 WG & 25 & 50 \\
\hline 2 & Flonicamida & Turbine 500 WG & 40 & 80 \\
\hline 3 & Flonicamida & Turbine 500 WG & 75 & 150 \\
\hline 4 & Thiamethoxam & Actara 250 WG & 100 & 200 \\
\hline 5 & Acetamiprido & Saurus 200 PS & 75 & 150 \\
\hline 6 & Imidacloprido & Provado 200 SC & 125 & 250 \\
\hline 7 & Testemunha & - & - & - \\
\hline
\end{tabular}


Para avaliar o efeito da exposição indireta de C. sanguinea a filme seco dos inseticidas, foram utilizados outros 40 tubos de vidro por tratamento. Neste caso, a aplicação dos inseticidas foi realizada antes da infestação e, uma hora após a aplicação, foi colocada em cada tubo uma joaninha C. sanguinea procedente da criação-estoque. Em seguida, cada tubo foi fechado com algodão e mantido em condições normais de temperatura e umidade em caixas porta-tubo. Diariamente, foram oferecidas presas (pulgões) às joaninhas.

As avaliações do efeito direto e indireto dos inseticidas sobre as joaninhas contidas nos tubos foram realizadas 14 horas e 1, 2, e 4 dias após a aplicação.

Experimento em casa-de-vegetaçãoe laboratório: Em casa-de-vegetação, foi semeada o cultivar Delta Opal em vasos de 3 L; após a germinação realizouse o desbaste, deixando-se apenas uma planta por vaso. Aos 30 dias após a germinação iniciou-se o experimento, adotando-se delineamento experimental de blocos ao acaso, com sete tratamentos e quatro repetições (Tabela 1 ).

Cada repetição foi composta por quatro gaiolas com dimensões de $15 \mathrm{~cm}$ de diâmetro por $30 \mathrm{~cm}$ de altura, revestidas com tecido tipo voil, onde em cada uma delas havia uma planta de algodão. Assim, cada tratamento consistia de 16 gaiolas (ou 16 plantas).

Com o intuito de avaliar o efeito da exposição direta dos inseticidas, foram colocadas no interior de cada gaiola 4 joaninhas $C$. saguinea procedentes da criação-estoque. Na sequência, foi realizada a aplicação dos inseticidas.

Aproximadamente 1 hora após a aplicação, colocaram-seno interior de cada gaiola outras 4 joaninhas C. saguinea, procedentes da criação-estoque, acondicionadas em saquinhos de filó (voil) devidamente identificados, com o objetivo de avaliar o efeito da aplicação indireta dos inseticidas.

A aplicação dos produtosfoi realizada em casa-devegetação com auxilio de pulverizador costal pressurizado $\left(\mathrm{CO}_{2}\right)$ à pressão de $40 \mathrm{lb} / \mathrm{pol}^{2}$, utilizando-se de ponta hidráulica modelo TLX3 e volume de calda de 200 L/ha. Após a aplicação, as gaiolas foram encaminhadas ao laboratório onde permaneceram em bancadas sob condições naturais de temperatura e umidade e, quando necessário, foram oferecidas presas (pulgões) às joaninhas.

Avaliou-se a sobrevivência de $C$. saguinea contidas nas gaiolas 1, 2, 3, 5 e 7 dias após a aplicação dos produtos.

Experimento em campo: Foi conduzido de 11 a 18/02/2005 em área pertencente à Estação Experimental da APTA. Utilizaram plantas do cultivar ${ }^{\prime}$ DeltaOpal' com 30 dias após a germinação. A princípio, foi realizado um levantamento populacional para determinação do nível de infestação de pulgões $A$. gossypii na área demarcada para instalação do ex- perimento. Após o levantamento, verificou-se que a infestação de pulgões encontrava-se uniforme em toda a área experimental.

Adotou-se delineamento em blocos ao acaso, com 4 repetições, sendo que cada repetição foi composta por uma parcela $(10 \times 10 \mathrm{~m})$ identificada por estacas de madeira.

Em cada parcela, colocou-se 4 saquinhos de filó de voil $(15 \times 30 \mathrm{~cm})$, contendo 4 joaninhas $C$. sanguinea cada um, procedentes da criação-estoque. Cada saquinho foi colocado no ponteiro de uma planta de algodão, de forma que revestisse o ponteiro da planta. Os saquinhos foram amarrados para evitar a fuga das joaninhas. Logo após a colocação dos saquinhos nas parcelas, procedeu-se a aplicação dos produtos conforme estabelecido na Tabela 1.

Da mesma forma, visando avaliar o efeito da aplicação indireta dos produtos sobre C. sanguinea, aproximadamente uma hora após a aplicação, foram colocados outros 4 saquinhos de filó de voil $(15$ × 30 $\mathrm{cm})$ por parcela, com cores diferentes daqueles utilizados na avaliação do efeito direto, sendo que cada saquinho continha outras 4 joaninhas $C$. sanguinea procedentes da criação-estoque.

A aplicação dos produtos foi realizada com pulverizador costal pressurizado $\left(\mathrm{CO}_{2}\right)$ a pressão de 40 $\mathrm{lb} / \mathrm{pol}^{2}$, utilizando-se de ponta hidráulica modelo TLX3 e volume de calda de $200 \mathrm{~L} / \mathrm{ha}$.

As avaliações de sobrevivência das joaninhas $C$. sanguinea contidas nos saquinhos de filó foram realizadas 1, 3, 5, e 7 dias após a aplicação. Avaliou-se também a eficiência dos produtos sobre o pulgão $A$. gossypii, realizando-se a contagem de pulgões vivos presentes em 25 plantas inteiras por parcela, 1, 4 e 7 dias após a aplicação dos produtos.

Os dados obtidos nos experimentos foram submetidos à análise de variância com auxílio de programa computacional (ESTAT). As médias foram comparadas pelo teste de Tukey, a 5\% de probabilidade e, para calcular a porcentagem de redução de joaninhas e de pulgões, utilizou-se a fórmula proposta por HENDERSON; TILTON (1955).

\section{RESULTADOS E DISCUSSÃO}

Experimento em laboratório: Pode-se observar na Tabela 2 que até 1 dia após a aplicação (DAA) não houve diferenças estatísticas entre os tratamentos e testemunha, com relação efeito da aplicação direta dos produtos sobre $C$. sanguinea.

A partir da avaliação realizada aos 2DAA, os tratamentos thiametoxam e imidacloprido diferiram da testemunha, evidenciando maior toxicidade desses produtos sobre C. sanguinea por ação direta. Embora os tratamentos à base de flonicamid e acetamiprido não tenham diferido da testemunha, verificou-se 
que também não diferiram estatisticamente dos tratamentos thiametoxam e imidacloprido em todas as avaliações realizadas.

Em contra partida, analisando os resultados para o efeito da exposição indireta aos produtos, foi possível constatar que em todas as avaliações não ocorreram diferenças estatísticas entre os tratamentos e a testemunha, ou seja, os inseticidas nas dosagens avaliadas acarretaram baixa mortalidade de C. sanguinea por exposição indireta. Os resultados obtidos concordaram com BACCI et al. (2006) que ressaltaram que a aplicação dos agrotóxicos, sempre que possível, deve ser realizada de forma seletiva, evitando a exposição direta dos inimigos naturais aos agrotóxicos e, assim, contribuindo para o controle biológico natural nos sistemas agrícolas.

Experimento em casa-de-vegetação e laboratório: Na Tabela 3 são apresentados os resultados do número médio de joaninhas $C$. sanguinea sobreviventes, tanto por efeito direto como indireto. Verificou-se que não houve diferenças estatísticas entre os tratamentos e a testemunha para o efeito da exposição indireta de C. sanguinea aos inseticidas, resultados semelhantes aos obtidos no experimento realizado em tubos de ensaio.

Tabela 2 - Número médio de joaninhas C. sanguinea sobreviventes nos tubos de ensaio, relativo às avaliações realizadas 14 horas; 1, 3, 5 e 7 dias após as aplicações dos produtos no experimento realizado em laboratório. Ribeirão Preto, SP.

\begin{tabular}{|c|c|c|c|c|c|c|c|c|c|}
\hline \multirow{3}{*}{ Tratamento } & \multirow{3}{*}{$\begin{array}{c}\text { Dosagens } \\
\text { g ou mL p. } \\
\text { c./ha }\end{array}$} & \multicolumn{4}{|c|}{ Efeito direto $^{1}$} & \multicolumn{4}{|c|}{ Efeito indireto $^{2}$} \\
\hline & & \multicolumn{4}{|c|}{ Avaliações } & \multicolumn{4}{|c|}{ Avaliações } \\
\hline & & 14 horas & $1 \mathrm{DAA}$ & $2 \mathrm{DAA}$ & $4 \mathrm{DAA}$ & 14 horas & $1 \mathrm{DAA}$ & 2 DAA & $4 \mathrm{DAA}$ \\
\hline Flonicamida & 50 & $7 \mathrm{a}$ & $7 \mathrm{a}$ & $6 a b$ & $5 \mathrm{ab}$ & $10 \mathrm{a}$ & $10 \mathrm{a}$ & $8 a$ & $7 \mathrm{a}$ \\
\hline Flonicamida & 80 & $7 \mathrm{a}$ & $7 \mathrm{a}$ & $6 \mathrm{ab}$ & $4 \mathrm{ab}$ & $10 \mathrm{a}$ & $10 \mathrm{a}$ & $8 \mathrm{a}$ & $7 \mathrm{a}$ \\
\hline Flonicamida & 150 & $6 a$ & $5 \mathrm{a}$ & $5 a b$ & $4 \mathrm{ab}$ & $10 \mathrm{a}$ & $10 \mathrm{a}$ & $7 \mathrm{a}$ & $6 a$ \\
\hline Thiamethoxam & 200 & $6 \mathrm{a}$ & $5 \mathrm{a}$ & $4 \mathrm{~b}$ & $2 \mathrm{~b}$ & $9 a$ & $7 a$ & $7 \mathrm{a}$ & $4 \mathrm{a}$ \\
\hline Acetamiprido & 150 & $7 \mathrm{a}$ & $9 \mathrm{a}$ & $6 \mathrm{ab}$ & $4 \mathrm{ab}$ & $10 \mathrm{a}$ & $10 \mathrm{a}$ & $7 \mathrm{a}$ & $6 a$ \\
\hline Imidacloprido & 250 & 8 a & $8 \mathrm{a}$ & $4 \mathrm{~b}$ & $2 b$ & $9 a$ & $7 \mathrm{a}$ & $7 \mathrm{a}$ & $5 a$ \\
\hline Testemunha & - & $10 \mathrm{a}$ & $10 \mathrm{a}$ & $9 \mathrm{a}$ & $7 \mathrm{a}$ & $10 \mathrm{a}$ & $10 \mathrm{a}$ & $10 \mathrm{a}$ & $10 \mathrm{a}$ \\
\hline \multicolumn{2}{|c|}{ C.V. $(\%)$} & 8,9 & 12,55 & 14,39 & 15,47 & 10,55 & 10,77 & 18,43 & 17,22 \\
\hline \multicolumn{2}{|c|}{ Teste F } & $1,27^{\mathrm{ns}}$ & $1,88^{*}$ & $8,04^{* *}$ & 9,68 ** & $1,96^{\mathrm{ns}}$ & $1,91^{\mathrm{ns}}$ & $2,34^{\mathrm{ns}}$ & $2,18^{\text {ns }}$ \\
\hline
\end{tabular}

Joaninhas C. sanguinea colocadas em tubos de ensaio antes da aplicação dos produtos.

${ }^{2}$ Joaninhas C. sanguinea colocadas em tubos de ensaio após a aplicação dos produtos.

DAA = dias após a aplicação.

*Médias seguidas por letras iguais na mesma coluna não diferem entre si, pelo teste de Tukey, a 5\% de probabilidade.

**Significativo pelo teste $\mathrm{F}$, a 5\% de probabilidade. ns - não significativo.

Tabela 3 - Número médio de joaninhas C. sanguinea sobreviventes, relativo às avaliações realizadas 1, 2, 3, 5 e 7 dias após a aplicação no experimento realizado em casa-de-vegetação e laboratório.

\begin{tabular}{|c|c|c|c|c|c|c|c|c|c|c|c|}
\hline \multirow{3}{*}{ Tratamentos } & \multirow{3}{*}{$\begin{array}{c}\text { Dosagens } \\
\text { g ou mL p. } \\
\text { c./ha }\end{array}$} & \multirow{2}{*}{\multicolumn{5}{|c|}{$\begin{array}{c}\text { Efeito direto }^{1} \\
\text { Dias após a aplicação }\end{array}$}} & \multirow{2}{*}{\multicolumn{5}{|c|}{$\begin{array}{c}\text { Efeito indireto }^{2} \\
\text { Dias após a aplicação }\end{array}$}} \\
\hline & & & & & & & & & & & \\
\hline & & 1 & 2 & 3 & 5 & 7 & 1 & 2 & 3 & 5 & 7 \\
\hline Flonicamida & 50 & $15 \mathrm{a}$ & $12 \mathrm{a}$ & $12 \mathrm{a}$ & $11 \mathrm{a}$ & $9 \mathrm{~b}$ & $14 \mathrm{a}$ & $12 \mathrm{a}$ & $10 \mathrm{a}$ & $9 \mathrm{a}$ & $9 \mathrm{a}$ \\
\hline Flonicamida & 80 & $14 \mathrm{a}$ & $12 \mathrm{a}$ & $9 \mathrm{ab}$ & $8 \mathrm{ab}$ & $8 \mathrm{~b}$ & $14 \mathrm{a}$ & $12 \mathrm{a}$ & $10 \mathrm{a}$ & $9 \mathrm{a}$ & $8 \mathrm{a}$ \\
\hline Flonicamida & 150 & $12 \mathrm{a}$ & $10 \mathrm{ab}$ & $8 \mathrm{ab}$ & $7 \mathrm{ab}$ & $6 \mathrm{bc}$ & $14 \mathrm{a}$ & $12 \mathrm{a}$ & $10 \mathrm{a}$ & $10 \mathrm{a}$ & $9 \mathrm{a}$ \\
\hline Thiamethoxam & 200 & $11 \mathrm{a}$ & $5 \mathrm{~b}$ & $4 \mathrm{~b}$ & $3 \mathrm{~b}$ & $0 \mathrm{c}$ & $11 \mathrm{a}$ & $8 \mathrm{a}$ & $7 \mathrm{a}$ & $3 a$ & $3 a$ \\
\hline Acetamiprido & 150 & $10 \mathrm{a}$ & $6 \mathrm{~b}$ & $2 \mathrm{~b}$ & $1 \mathrm{~b}$ & $0 \mathrm{c}$ & $12 \mathrm{a}$ & $10 \mathrm{a}$ & $6 a$ & $6 a$ & $6 a$ \\
\hline Imidacloprido & 250 & $10 \mathrm{a}$ & $4 \mathrm{~b}$ & $2 b$ & $1 \mathrm{~b}$ & $0 \mathrm{c}$ & $13 \mathrm{a}$ & $10 \mathrm{a}$ & $6 a$ & $3 a$ & $2 a$ \\
\hline Testemunha & - & $16 \mathrm{a}$ & $15 \mathrm{a}$ & $15 \mathrm{a}$ & $15 \mathrm{a}$ & $14 \mathrm{a}$ & $15 \mathrm{a}$ & $14 \mathrm{a}$ & $12 \mathrm{a}$ & $10 \mathrm{a}$ & $9 a$ \\
\hline C.V. $\left({ }^{\circ}\right.$ & & 21,14 & 18,47 & 25,71 & 29,01 & 30,23 & 11,19 & 12,34 & 15,97 & 29,01 & 35,34 \\
\hline Teste & & $1,99^{\text {ns }}$ & $8,34^{* *}$ & $7,79 * *$ & $9,78^{* *}$ & $12,45^{\mathrm{ns}}$ & $2,39^{\text {ns }}$ & $1,81^{\mathrm{ns}}$ & $1,74^{\mathrm{ns}}$ & $1,67^{\mathrm{ns}}$ & $1,03^{\text {ns }}$ \\
\hline
\end{tabular}

${ }^{1}$ Joaninhas C. sanguinea acondicionadas em saquinhos de filó e colocadas nas gaiolas antes da aplicação dos produtos. ${ }_{2}^{2}$ Joaninhas C. sanguinea acondicionadas em saquinhos de filó e colocadas nas gaiolas após a aplicação dos produtos. Médias seguidas por letras iguais na mesma coluna não diferem entre si, pelo teste de Tukey, a 5\% de probabilidade. **Significativo pelo teste $\mathrm{F}$, a $5 \%$ de probabilidade. ns - não significativo. 
Porém, a partir da avaliação realizada aos 2 DAA para avaliação do efeito direto dos inseticidas, foram observadas diferenças entre os tratamentos com a relação à sobrevivência de $C$. sanguinea. Com base nos resultados, pode-se inferir que os tratamentos thiamethoxam, acetamiprido e imidacloprido apresentaram comportamento semelhante sobre C. sanguinea, pois, nas avaliações de sobrevivência, realizadas a partir dos 2 DAA, estes tratamentos diferiram da testemunha, mas não entre si.

Os tratamentos com flonicamida não diferiram da testemunha até a avaliação realizada aos $5 \mathrm{DAA}$, evidenciando menor toxicidade desse ingrediente ativo a C. sanguinea. Resultados semelhantes foram obtidos por NogueIraet al. (2007) que avaliaram a seletividade dediversosinseticidas aos principaisinimigos naturais de pragas do algodoeiro em condições de campo e concluíram que, dentre os inseticidas utilizados, o flonicamida apresentou a menor mortalidade de coccinelídeos, sendo considerado pelos autores como seletivo aos inimigos naturais.

Experimento em campo: Constatou-se que todos os tratamentos mostraram-se altamente eficientes no controle do pulgão A. gossypii, com percentuais de redução superior a 79\%, nas avaliações realizadas 1 , 4 e 7 DAA. Segundo Corso et al. (1999), a eficiência de controle de pragas em torno de $80 \%$ representou a melhor relação entre a necessidade de controle e a preservação de inimigos naturais, haja vista que quanto melhor o controle de pulgões, menor a presença dos seus predadores, devido a menor quantidade de presas disponíveis.

De maneira geral, constatou-se que a sobrevivência de C. sanguinea em campo foi menor do que em laboratório. Segundo Gould et al. (1991), no campo, além das intempéries ocorridas, a eliminação das presas após a aplicação de um determinado agrotóxico contribuiu para o aumento da mortalidade de inimigos naturais devido à falta de alimento.
Nas avaliações realizadas aos 5 e 7 DAA, os tratamentos thiamethoxam, acetamiprido e imidacloprido apresentaram sobrevivência de C. sanguinea inferior aos tratamentos com flonicamida e diferiram estatisticamente da testemunha, mas não entre si. Todavia, SCARPELLINI (2008) ressaltou que o inseticida thiamethoxan, embora reduza a população de joaninhas C. sanguinea, apresenta maior seletividade que os inseticidas carbosulfano e imidacloprido. De acordo com o autor, a seletividade dos inseticidas facilita a recuperação da população de inimigos naturais importantes no controle biológico na cultura do algodoeiro.

O efeito dos produtos sobre $C$. sanguinea, avaliado em condições de campo, pode ser observado na Tabela 4. Por efeito direto, observou-se que, na primeira avaliação realizada $1 \mathrm{DAA}$, não houve diferenças entre os tratamentos e a testemunha. Contudo, a partir da segunda avaliação, realizada aos 3 DAA, todos os tratamentos diferiram da testemunha, mas não dos tratamentos que se empregou flonicamida. Nas avaliações realizadas aos 5 e 7 DAA, evidenciou-se uma maior sobrevivência de C. sanguinea, quando comparado aos demais inseticidas.

De acordo com CASIDA; QUISTAD (1998), o inseticida flonicamida aparentemente é capaz de atuar por um novo mecanismo de ação sobre o sistema nervoso dos insetos, que ainda não foi totalmente elucidado, o que pode explicar parcialmente essa maior seletividade a $C$. sanguinea.

Os resultados obtidos devido à aplicação indireta foram semelhantes aos obtidos com a aplicação direta, ou seja, dentre os inseticidas avaliados, os tratamentos à base de flonicamida apresentaram a maior sobrevivência de $C$. sanguinea, em contrapartida, thiamethoxam, acetamiprido e imidacloprido acarretaram em menor sobrevivência de $C$. sanguinea.

Tabela 4 - Número médio de joaninhas C. sanguinea sobreviventes relativo às avaliações realizadas 1, 3, 5 e 7 dias após as aplicações dos produtos nos experimento realizado em campo. Ribeirão Preto, SP.

\begin{tabular}{|c|c|c|c|c|c|c|c|c|c|}
\hline \multirow{3}{*}{ Tratamento } & \multirow{3}{*}{$\begin{array}{c}\text { Dosagens g } \\
\text { ou mL p.c./ha }\end{array}$} & \multicolumn{4}{|c|}{ Efeito direto $^{1}$} & \multicolumn{4}{|c|}{ Efeito indireto $^{2}$} \\
\hline & & \multicolumn{4}{|c|}{ Dias após a aplicação } & \multicolumn{4}{|c|}{ Dias após a aplicação } \\
\hline & & 1 & 3 & 5 & 7 & 1 & 3 & 5 & 7 \\
\hline Flonicamida & 50 & $12 \mathrm{a}$ & $8 \mathrm{~b}$ & $7 \mathrm{~b}$ & $5 \mathrm{~b}$ & $13 \mathrm{a}$ & $9 \mathrm{ab}$ & $8 \mathrm{a}$ & $6 \mathrm{ab}$ \\
\hline Flonicamida & 80 & $11 \mathrm{a}$ & $7 \mathrm{~b}$ & $7 \mathrm{~b}$ & $5 \mathrm{~b}$ & $12 \mathrm{a}$ & $9 \mathrm{ab}$ & $8 \mathrm{a}$ & $6 \mathrm{ab}$ \\
\hline Flonicamida & 150 & $11 \mathrm{a}$ & $7 \mathrm{~b}$ & $6 \mathrm{~b}$ & $4 \mathrm{~b}$ & $12 \mathrm{a}$ & $7 \mathrm{ab}$ & $6 \mathrm{a}$ & $4 \mathrm{~b}$ \\
\hline Thiamethoxam & 200 & $10 \mathrm{a}$ & $7 \mathrm{~b}$ & $2 c$ & $0 \mathrm{c}$ & $10 \mathrm{a}$ & $3 \mathrm{~b}$ & $0 \mathrm{~b}$ & $0 \mathrm{c}$ \\
\hline Acetamiprido & 150 & $9 \mathrm{a}$ & $5 c$ & $1 \mathrm{c}$ & $0 \mathrm{c}$ & $10 \mathrm{a}$ & $2 b$ & $0 \mathrm{~b}$ & $0 \mathrm{c}$ \\
\hline Imidacloprido & 250 & $9 \mathrm{a}$ & $5 c$ & $2 c$ & $0 \mathrm{c}$ & $10 \mathrm{a}$ & $3 b$ & $0 \mathrm{~b}$ & $0 \mathrm{c}$ \\
\hline Testemunha & - & $13 \mathrm{a}$ & $12 \mathrm{a}$ & $10 \mathrm{a}$ & $9 \mathrm{a}$ & $15 \mathrm{a}$ & $14 \mathrm{a}$ & $11 \mathrm{a}$ & $10 \mathrm{a}$ \\
\hline C.V. $(\%)$ & & 22,35 & 19,88 & 26,56 & 30,66 & 27,35 & 28,44 & 29,01 & 29,23 \\
\hline Teste F & & $1,95^{\mathrm{ns}}$ & $12,44^{\star *}$ & $11,07^{\star *}$ & $13,13^{* *}$ & $1,34^{\mathrm{ns}}$ & $5,93^{*}$ & $7,66^{* *}$ & $10,56^{* *}$ \\
\hline
\end{tabular}

1Joaninhas C. sanguinea acondicionadas em saquinhos de filó e colocadas nas parcelas antes da aplicação dos produtos.

${ }^{2}$ Joaninhas C. sanguinea acondicionadas em saquinhos de filó e colocadas nas parcelas após a aplicação dos produtos. Médias seguidas por letras iguais na mesma coluna não diferem entre si, pelo teste de Tukey, a 5\% de probabilidade. **Significativo pelo teste $\mathrm{F}$, a $5 \%$ de probabilidade. ns - não significativo. 
Na Figura 1 são apresentados os resultados obtidos para os diferentes tratamentos em relação à porcentagem de redução de $C$. sanguinea em condições de campo, tanto por efeito direto com indireto.

De maneira geral, pode-se observar que a porcentagem de redução aumentou à medida que aumentou o tempo de exposição das joaninhas ao resíduo dos produtos. Por efeito direto e indireto, decorridos $7 \mathrm{DAA}$, verificou-se que os tratamentos thiamethoxam, acetamiprido e imidacloprido apresentaram percentuais de redução de C. sanguinea de $100 \%$ e os tratamentos à base de flonicamida obtiveram percentuais que variaram de 40 a $60 \%$.

Diante do exposto, torna-se evidente a importância do uso de produtos seletivos na preservação e incremento das populações de artrópodes predadores, bem como na diminuição do impacto ambiental e dos custos de produção. Segundo CARVALHO et al. (2001), a associação entre os métodos químico e biológico no controle de pragas é fundamental para garantir a sustentabilidade do sistema agrícola.

Tabela 5 - Número médio de pulgões A. gossypii encontrados por tratamento no experimento de campo, nas avaliações realizadas aos 1, 4 e 7 dias após o tratamento (DAT). Teste de Tukey a 5\% de probabilidade e porcentagem de redução populacional, em relação à testemunha. Ribeirão Preto, SP.

\begin{tabular}{|c|c|c|c|c|c|c|c|c|c|c|c|}
\hline \multicolumn{3}{|c|}{ Tratamentos } & \multicolumn{9}{|c|}{ Avaliações } \\
\hline $\mathrm{N}^{\mathrm{o}}$ & Produtos & g ou mL p.c./ha & \multicolumn{3}{|c|}{$1 \mathrm{DAT}$} & \multicolumn{3}{|c|}{$4 \mathrm{DAT}$} & \multicolumn{3}{|c|}{$7 \mathrm{DAT}$} \\
\hline 1 & Flonicamida 500 WG & 50 & $295^{1}$ & $\mathrm{~b}$ & 80 & 2391 & $\mathrm{~b}$ & 89 & $218^{1}$ & $\mathrm{~b}$ & 89 \\
\hline 2 & Flonicamida 500 WG & 80 & 198 & $b$ & 87 & 91 & $b$ & 96 & 119 & $b$ & 94 \\
\hline 3 & Flonicamida 500 WG & 150 & 163 & $b$ & 89 & 25 & $b$ & 99 & 91 & $b$ & 95 \\
\hline 4 & Tiametoxam 250 WG & 200 & 172 & $b$ & 88 & 84 & $b$ & 96 & 75 & $b$ & 96 \\
\hline 5 & Acetamiprido 200 SP & 150 & 181 & $b$ & 88 & 70 & $\mathrm{~b}$ & 97 & 86 & $b$ & 95 \\
\hline 6 & Imidacloprido 200 SC & 250 & 189 & $\mathrm{~b}$ & 88 & 88 & $\mathrm{~b}$ & 96 & 92 & $\mathrm{~b}$ & 95 \\
\hline 7 & Testemunha & ------- & 1470 & $\mathrm{a}$ & - & 2100 & $\mathrm{a}$ & - & 1900 & $\mathrm{a}$ & - \\
\hline \multicolumn{3}{|c|}{ C.V. $(\%)$} & \multicolumn{3}{|c|}{21,45} & \multicolumn{3}{|c|}{27,93} & \multicolumn{3}{|c|}{25,67} \\
\hline \multicolumn{3}{|c|}{ Teste F } & \multicolumn{3}{|c|}{$24,67^{* *}$} & \multicolumn{3}{|c|}{$24,67^{* *}$} & \multicolumn{3}{|c|}{$24,67^{* *}$} \\
\hline
\end{tabular}

Médias seguidas por letras iguais na mesma coluna não diferem entre si, pelo teste de Tukey, a 5\% de probabilidade. **Significativo pelo teste $\mathrm{F}$, a $5 \%$ de probabilidade. ns - não significativo.

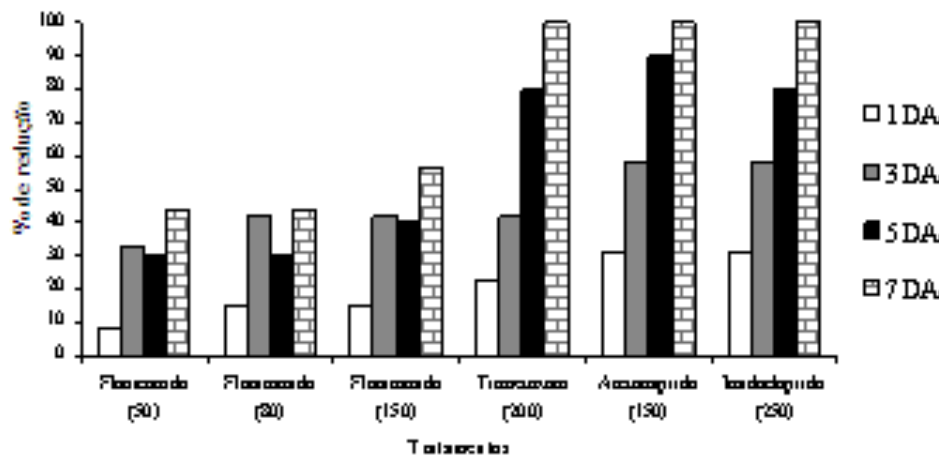

A

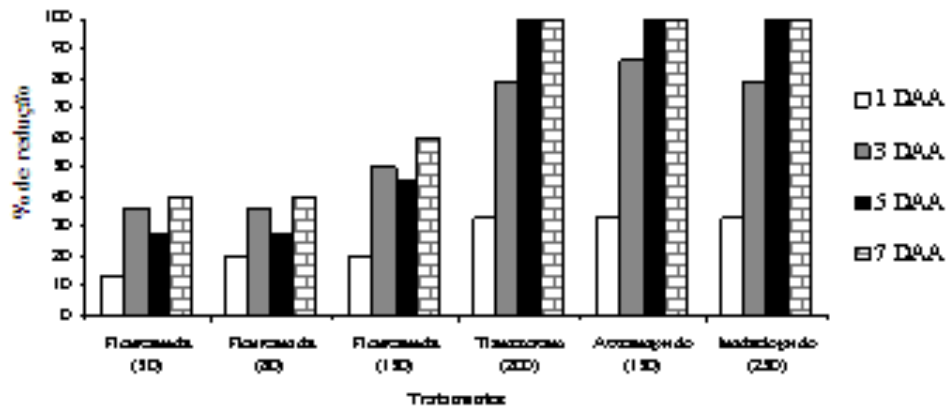

B

Fig. 1. Porcentagem de redução de joaninhas C. sanguinea nos diferentes tratamentos expressos em $g$ ou $\mathrm{mL}$ de p.c/ha, nas avaliações realizadas aos 1, 3, 5 e 7 dias após a aplicação (DAA) no experimento de campo. (A) efeito direto; (B) efeito indireto. 


\section{CONCLUSÕES}

-O inseticida flonicamida apresentou maior seletividade à joaninha predadora C. sanguinea em relação aos demais inseticidas;

- Os tratamentos thiamethoxam, acetamiprido e imidacloprido apresentaram sobrevivência de $C$. sanguinea inferior aos tratamentos com flonicamida; - Os tratamentos thiamethoxam, acetamiprido e imidacloprido apresentaram comportamento semelhantes;

- Todos os inseticidas avaliados mostraram-se altamente eficientes no controle do pulgão do algodoeiro A. gossypii em campo.

\section{AGRADECIMENTOS}

Os autores agradecem ao Dr. Luis Cláudio Paterno Silveira (UFLA - Lavras) pela identificação dos pulgões e Giuliano Roberto Scarpellini, pela manutenção das joaninhas em laboratório.

\section{REFERÊNCIAS}

BACCI, L.; PEREIRA, E.J.G.; FERNANDES, F.L.; PICANÇO, M.C.; CRESPO, A.L.B.; CAMPOS, M.R. Seletividade Fisiológica de Inseticidas a Vespas Predadoras (Hymenoptera: Vespidae) de Leucoptera coffeella (Lepidoptera: Lyonetiidae). Revista Bioassay, v.10, n.1, p.1-7, 2006. (Online).

BOIÇA JUNIOR, A.L.; SANTOS, T.M.; KURANISHI, A.K. Desenvolvimento larval e capacidade predatória de Cycloneda sanguinea (L.) e Hippodamia convergens Guerin-Men. alimentadas com Aphis gossypii Glover sobre cultivares de algodoeiro. Acta Scientiarum Agronomy, v.26, p.239-244, 2004.

CARVALHO, G.A.; PARRA, J.R.P.; BATISTA, G.C. Seletividade de alguns produtos fitossanitários a duas linhagens de Trichogramma pretiosum Riley, 1879 (Hymenoptera: Trichogrammatidade). Ciência e Agrotecnologia, v.25, n.3, p.583-591, 2001.

CASIDA, J.E.; QUISTAD, G.B. Gold age of insecticide research: past, presente, or future. Annual Review of Entomology, v.43, p.1-16, 1998.

CORSO, I.C.; GAZZONI, D.L.; NERY, M.E. Efeito de doses de refúgio sobre a seletividade de inseticidas a predadores e parasitóides de pragas da soja. Pesquisa Agropecuária Brasileira, v.34, p.1529-1538, 1999.

GOULD, F.; KENNEDY, G.G.; JOHNSON, M.T. Effects of natural enemies on the rate of herbivore adaptation to resistant host plants. Entomologia Experimentalis et Applicata, v.58, n.1, p.1-14, 1991.

HASSAN, S.A.; BIGLER, F.; BOGENSCHUTZ, H.; BOLLER, E.; BRUN, J.; CALIS, J.N.M.; COREMANSPELSENEER, J.; DUSO, C.; GROVE, A.; HEINBACH, U.J.; HELYER, N; HOKKAMEN, H.; LEWIS, G.B.; MANSOUR, F.; MORETH, L.; POLGAR, L; SANSOEPETERSEN, L.; SAUPHANOR, STAUBLI, B.A.; STERK, G.; VAINIO, A.; VAN DE VEIRE, M.; VIGGIANI, G.; VOGHT, H. Results of the sixth joint pesticide testing programme of the IOBC/WPRS Working Group "Pesticides and Beneficial Organisms". Entomophaga, v.39, p.107-119, 1994.

HENDERSON, C.F.;TILTON, E.W. Tests with acaricides against the brown wheat mite. Journal Economic Entomology, v.48, p.157-161, 1955.

HODEK, I. Bionomics and ecology of predaceous Coccinellidae. Annual Review of Entomology, v.12, p.79-104, 1967.

ISIKBER, A.A.; COPLAND, M.J.W. Effects of various aphid foods on Cycloneda sanguinea. Entomologia Experimental et Applicata, v.102, n.1, p.93-97, 2002.

MAIA, V.B.; BUSOLI, A.C.; DELABIE, J.H.C. Seletividade fisiológica de Endossulfan e Deltametrina às operárias de A. chartifex spiriti For. (Hymenoptera, Formicidae) em Agroecossistema cacaueiro do Sudeste da Bahia. Neotropical Entomology, v.30, n.2, p.449-454, 2001.

NOGUEIRA, R.F.; LIMA JR, I.S. de; BERTONCELLO, T.F.; MELO, E.P. de; SUEKANE, R.; DEGRANDE, P.E. Seletividade de inseticidas aos inimigos naturais das pragas do algodoeiro. In: CONGRESSO BRASILEIRO DE ALGODÃO, 6., 2007, Uberlândia, MG. Anais. Uberlândia, 2007. CD-Rom.

SANTOS, W.J. Pragas do algodoeiro. In: Mato Grosso Liderança e Competitividade. Rondonópolis: Fundação MT/Embrapa, 1999. p.113-149. (Boletim, 3).

SCARPELLINI, J.R. Seletividade fisiológica de aficidas sobre joaninha Cycloneda sanguinea (Linnaeus, 1763) (Coleoptera, Coccinellidae) em algodoeiro. Arquivos do Instituto Biológico, São Paulo, v.75, n.2, p.195-202, 2008.

SOARES, J.J.; BUSOLI, A.C. Efeito de inseticidas em insetos predadores em culturas de algodão. Pesquisa Agropecuária Brasileira, v.35, n.9, p.1889-1894, 2000.

Recebido em 27/1/10

Aceito em19/4/11 